\title{
ENDOR study of irradiated tooth enamel
}

\author{
S. Ishchenko, I. Vorona, S. Okulov \\ Institute of Semiconductor Physics, National Academy of Sciences of Ukraine, Kyiv, 252028, Ukraine
}

\begin{abstract}
EPR and ENDOR. Radiationinduced EPR spectrum of tooth enamel was found to be a superposition of signals with dominant contribution determined by $\mathrm{CO}_{2}^{-}$radicals. Two types of these radicals were observed: ordered and disordered centers. EPR spectra of both $\mathrm{CO}_{2}^{-}$centers are described by axial $g$-tensor with $g_{\|}=1.9975$ and $g \perp=2.0021$ with $g_{\|} \| c$. The ENDOR spectrum of unannealed enamel powder consists of a singlet at Larmor frequency of ${ }^{31} \mathrm{P}$ nuclei and doublet at Larmor frequency of ${ }^{1} \mathrm{H}$ nuclei. Samples annealing at $\mathrm{T}=200-250^{\circ} \mathrm{C}$ resulted in the destruction of disordered centers and appearence of superhyperfine structure of ENDOR spectra. Its analysis with advanced the powder ENDOR theory allows to determine the superhyperfine constants and to find for the first time that the ordered $\mathrm{CO}_{2}^{-}$radical is located in $\mathrm{B}$ sites (phosphorous position) of bioapatite lattice. This substitution is accompanied by the shift of the nuclei of the first ${ }^{31} \mathrm{P}$ shell towards the defects by $0.04 \mathrm{~nm}$ and the formation of the $\mathrm{OH}$ vacancy in the nearest radical surroundings.
\end{abstract}

Keywords: tooth enamel, electron-nuclear double resonance, radiation-induced electron-paramagnetic resonance.

Paper received 11.12.98; revised manuscript received 01.04.99; accepted for publication 19.04.99.

\section{Introduction}

The investigation of bioapatites has been started a few decades ago. Late in the 60 s apatites have been considered as a perspective laser material and studied by different techniques. Radiospectroscopic investigations have been directed to clarification of mechanisms of incorporation of impurity atoms such as carbon, silicon, sulphur and different cations in apatites lattice. The complex study of naturae, synthetic and bioapatites has resulted in building up of laser operating in IR region [ 1]. Besides, calcium apatites had attracted considerable attention because they form a base of the important biological tissues such as bone and dental enamel. In these tissues crystallites of hydroxyapatite are organized into small prisms plunged into organic substance. Both prisms and crystallites reveal certain ordering. The composition and orientation of crystallites are believed to be closely related to the total state of organism's health. Therefore, the information about their changes can be used in medicine for early diagnostics of some diseases [2]. Besides, ionizing irradiation of bioapatites induces paramagnet- ic centers, the number of which is proportional to the irradiation dose. This phenomenon is used in retrospective dosimetry to determine radiation dose received by people and animals [3-5] and in EPR dating of archeological findings $[6,7]$.

Tooth enamel is the most interesting calcified biological tissue. It contains a mineral phase representing about $94-98 \%$ of the total weight, the rest is formed by water and organic matter. The EPR signal near $g=2.0$ appeared after irradiation by $\gamma$ - and $x$-rays is well known [8-14]. However, the nature of this radiation-induced signal is not clarified completely in spite of many publications devoted to its study.

EPR spectrum in the irradiated tooth enamel has been found to be a composite signal [13]. It leads to variation of the line shape depending on experimental conditions and sample history. This is the reason of discrepancies among the data reported in different papers and leading to uncertainty and mistakes in EPR dosimetry.

The $\mathrm{CO}_{2}^{-}, \mathrm{CO}_{3}^{3-}, \mathrm{CO}_{3}^{-}, \mathrm{CO}^{-}$radicals [13], $\mathrm{O}^{-}$center [14] 


\section{S. Ishchenko et al.: ENDOR study of irradiated tooth enamel}

and the so-called "background signal" with $g=2.0045$, $\Delta H_{p p}=0.64 \mathrm{mT}$ give the main contribution to the mentioned radiation-induced EPR spectrum. The background signal is observed in the enamel samples before irradiation and dominates at irradiation doses up to 1 Gy. Note that radicals can be located in different sites of hydroxyapatite lattice resulting in the increase of the number of the EPR components. Besides, the irradiation of tooth enamel induces short-half-life radicals. Some centers are destroyed by temperature annealing whereas some new defects can be formed in accordance with annealing processing.

However, the contributions of the mentioned centers are not equivalent. A dominant contribution is caused by the centers of one type. Some authors have assigned this more intensive signal to $\mathrm{CO}_{3}{ }^{3-}$ radical $[8-10,15]$ while others have classified it as $\mathrm{CO}_{2}^{-}$radical. The most convincing evidence of $\mathrm{CO}_{2}^{-}$interpretation is the presence of g-tensor component equal to 1.997. This value is characteristic for $\mathrm{CO}_{2}^{-}$and varies slightly depending on radical surroundings. Besides, the recent study of $\mathrm{CO}_{3}{ }^{3-}$ in synthetic hydroxyapatite [16] allows to determine the following $g$ values of the radical: $g_{\mathrm{x}}=2.0045, g_{\mathrm{y}}=$ $=2.0034, g_{z}=2.0014$. These parameters do not describe the dominant EPR signal in tooth enamel.

Callens et al. [17] have carried out the EPR study of intact and powdered human tooth enamel both unheated and dried at $400{ }^{\circ} \mathrm{C}$. Existence of two main EPR components with similar parameters has been found. One of them does not exhibit anisotropy and determined by disordered centers. This component has been attributed to $\mathrm{CO}_{2}^{-}$radical in organic matrix of enamel and/or surface $\mathrm{CO}_{2}^{-}$radical. Another EPR component has been assigned to a bulk $\mathrm{CO}_{2}^{-}$radical. It has been noted that the annealing results in the destruction of the disordered centers. However, the position in the lattice has not been determined for both radicals.

ENDOR is the most useful technique to define the location of a paramagnetic center in crystalline lattice. A few ENDOR measurements were carried out on tooth enamel [8, 10, 18, 19]. The dominant ENDOR signals observed in these experiments were the structureless singlet line at Larmor frequency of ${ }^{31} \mathrm{P}$ nuclei and singlet or doublet line at Larmor frequency of ${ }^{1} \mathrm{H}$ nuclei. Such ENDOR spectra do not allow to obtain information about the location of $\mathrm{CO}_{2}^{-}$radical in hydroxyapatite lattice. More informative ENDOR results have been obtained on the synthetic hydroxyapatite. The superhyperfine structure of the ${ }^{31} \mathrm{P}$ and ${ }^{1} \mathrm{H}$ ENDOR spectra permits to determine the location of $\mathrm{CO}_{3}^{3-}[16]$ and $\mathrm{O}^{-}[14]$ radicals in this material.

This article is devoted to the detailed ENDOR study of irradiated tooth enamel to define the location of $\mathrm{CO}_{2}$ in hydroxyapatite crystallites. The structure of ${ }^{1} \mathrm{H}$ and ${ }^{31} \mathrm{P}$ ENDOR lines was observed on enamel samples dried at $250{ }^{\circ} \mathrm{C}$. Its analysis allowed to obtain the information about $\mathrm{CO}_{2}^{-}$surroundings and to determine the radical location in bioapatite lattice.

\section{Materials and methods}

The powder samples were prepared according to the traditional procedure of retrospective EPR dosimetry [3]. The paramagnetic centers were created by irradiation with $x$ - or $\gamma$ - rays. The absorption dose was estimated to be approximately $10 \mathrm{kGy}$. The heating of the samples was carried out on air at temperatures $250-300{ }^{\circ} \mathrm{C}$ during 30-60 minutes. Such annealing conditions were optimum to observe the superhyperfine structure of ENDOR spectra.

ENDOR spectra were recorded using EYa-1301 superheterodyne spectrometer operating in the $3-\mathrm{cm}$ range of wavelengths. Measurements were performed at the temperatures $77,4.2$ and $1.5 \mathrm{~K}$. A sample was placed along the axis of a $\mathrm{TE}_{011}$ cylindrical microwave cavity. A radiofrequency (rf) field inducing nuclear transitions was generated by four rods driven across the cavity parallel to its axis and connected in accordance with Helmholtz scheme. The amplitudes of rf field were 0.01-0.05 mT. The ENDOR signals were registrated by pulse modulation at $1.5 \mathrm{kHz}$ of the applied rf power and phase-sensitive detection. The ENDOR spectra were recorded for powder samples of the human and swine tooth enamel. On heated samples the structure of ENDOR lines was obtained with microwave power of approximately $5 \mu \mathrm{W}$ at liquid helium temperature. The dependence of ENDOR spectra on the type of irradiation ( $x$ - or $\gamma$ - rays) and type of enamel (human or swine) was not observed.

\section{The theory of powder endor}

The orientation dependencies of ENDOR spectra can be studied on powders if $g$-tensor of paramagnetic center is anisotropic. During ENDOR measurements the magnetic field $\mathrm{H}$ is fixed in certain position of a powder EPR spectrum. This induces the resonance only for those centers which have definite orientation relative to the direction of the magnetic field. The changing of $H$ value permits to observe the centers with different orientations separately, i.e., to registrate the angular variation of ENDOR lines. The obtained dependencies are similar to that of single crystals if superhyperfine A-tensor and electron $g$-tensor have axial symmetry and coinciding directions. In general case, ENDOR spectrum is partly averaged. However, the lines of such spectrum alter their spectral positions while changing the value of fixed magnetic field. This permits to obtain the information about the parameters of superhyperfine interaction. The above technique has been named "orientation ENDOR selection" $[20,21]$.

The general theory of powder ENDOR is very complicated. Its particular cases have been described before [21-24]. It is appropriate to present the simplified expressions which were used in this work. The expressions have been obtained in the first order of the perturbation theory and given the satisfactory description of our experimental results. Note that these expressions do 


\section{S. Ishchenko et al.: ENDOR study of irradiated tooth enamel}

not consider the effect of alternating fields, relaxation processes and transition probabilities. They contain also some limitations which will be pointed out below.

The spectrum of powder ENDOR caused by unpaired electron and nucleus with spins $S=I=1 / 2$ can be described in $g$-tensor axes frame (see Fig. 1) by the following expression:

$$
\begin{aligned}
& I(v, H)=c \int_{0}^{\pi / 2} \int_{0}^{2 \pi} \sin \theta \sum_{m} L\left(H-H_{0}^{m}(\theta, \varphi)\right) \times \\
& \times \sum_{M} N\left(v-v_{0}^{M}(H, \theta, \varphi)\right) d \theta d \varphi
\end{aligned}
$$

where $L\left(H-H_{0}\right)$ and $N\left(n-n_{0}\right)$ are the form-factors of EPR and ENDOR line shapes, respectively, $H_{0}$ and $v_{0}$ are the resonant field of EPR and resonant frequency of ENDOR, $M$ and $m$ are the projections of electron and nuclear spins on $H$ direction, $\mathrm{C}$ is a constant. In (1) $H$ is a parameter determining the point where EPR spectrum is saturated and, thus, the group of paramagnetic centers with the same definite orientation.

$\mathrm{H}_{0}$ and $\mathrm{V}_{0}$ can be obtained from the spin-Hamiltonian accounting for electron Zeeman, nuclear Zeeman and superhyperfine interactions $[21,25]$.

$$
H_{0}^{m}(\theta, \varphi)=\frac{h v_{m w}-m A(\theta, \varphi)}{\beta g(\theta, \varphi)},
$$

$$
\begin{aligned}
& v_{0}^{M}(H, \theta, \varphi)= \\
& =\left\{\sum_{i}\left[\frac{M}{h g(\theta, \varphi)}\left(\sum_{j} g_{j} h_{j} A_{i j}\right)-h_{i} v_{n}(H)\right]^{-2}\right\}^{1 / 2},
\end{aligned}
$$

where

$$
\begin{aligned}
& g(\theta, \varphi)=\left[\sum_{i}\left(g_{i} h_{i}\right)^{2}\right]^{1 / 2}, \\
& A(\theta, \varphi)=\left[\sum_{i}\left(\sum_{j} A_{i j} g_{j} h_{j}\right)^{2}\right]^{1 / 2} / g(\theta, \varphi),
\end{aligned}
$$

$v_{n}(H)=g_{n} \beta_{n} H / h$,

and $A_{i j}$ are the components of superhyperfine A-tensor in $g$-tensor axes frame, $h_{i}(i=1-3)$ denote the direction cosines of magnetic field vector, $g_{i}$ are the principal values of $g$ tensor, $g_{\mathrm{n}}$ is the nuclear $g$ factor, $\beta$ and $\beta_{n}$ are the Bohr magneton and nuclear magneton, respectively, $h$ is the Planck's constant, $v_{m \omega}$ is the microwave frequency of EPR spectrometer.

The expressions for resonant field (2) and resonant frequencies (3) contain many parameters and some simplifications can be made.

Superhyperfine tensor has been considered to have axial symmetry along the direction on nucleus, $\mathbf{r}_{n}$. Then, in $x^{\prime}, y^{\prime}, \quad z^{\prime}$ axes frame with $z^{\prime} \| \mathbf{r}_{n}$ it can be presented as following:

$A^{\prime}=\left[\begin{array}{ccc}a-b & 0 & 0 \\ 0 & a-b & 0 \\ 0 & 0 & a+2 b\end{array}\right]$

where

$a=\frac{1}{3} S p A^{\prime}$,

$b=\frac{1}{3}\left(A_{33^{\prime}}-A_{2^{\prime} 2^{\prime}}\right)$,

and $a$ is a Fermi constant of isotropic superhyperfine interaction, $b$ is a constant of dipole-dipole interaction determining the anisotropic superhyperfine interaction [25].

We shall restrict ourselves to the axial symmetry of $g$-tensor in EPR. In this case we can consider that $\varphi_{n}=0$ and $y^{\prime} \| y$. Then, the superhyperfine tensor in $g$-tensor axes frame can be written as:

$A=\left[\begin{array}{ccc}a+b\left(3 \sin ^{2} \theta_{n}-1\right) & 0 & 3 b \cos \theta_{n} \sin \theta_{n} \\ 0 & a-b & 0 \\ 3 b \cos \theta_{n} \sin \theta_{n} & 0 & a+b\left(3 \cos ^{2} \theta_{n}-1\right)\end{array}\right]$

Components $\mathrm{A}_{i j}$ of A-tensor and ones $\mathrm{A}_{i^{\prime}{ }^{\prime},}$ of $\mathrm{A}^{\prime}$-tensor are related by transformation of the axes frame. Superhyperfine interaction between $\mathrm{CO}_{2}^{-}$and surrounding nuclei is smaller than the EPR linewidth, therefore it can be neglected in (2). Besides, the anisotropy of $g$ tensor of $\mathrm{CO}_{2}^{-}$radical in tooth enamel is small [10] and $g$ can be considered as an isotropic value in (3). Therefore, the spectrum of powder ENDOR can be written as:

$$
\begin{aligned}
& I(v, H)=c^{\pi / 2} \int_{0}^{\pi} \int_{0} \sin \theta L\left(H-H_{0}(\theta)\right) \\
& {\left[N\left(v-v_{0}^{+}(H, \theta, \varphi)\right)+N\left(v-v_{0}^{-}(H, \theta, \varphi)\right)\right] d \theta d \varphi,}
\end{aligned}
$$

$H_{0}(\theta)=\frac{h v_{m \omega}}{\beta\left[g_{\perp}^{2}+\left(g_{\|}^{2}-g_{\perp}^{2}\right) \cos ^{2} \theta\right]^{1 / 2}}$,

$v_{0}^{ \pm}(H, \theta, \varphi)=$ 


\section{S. Ishchenko et al.: ENDOR study of irradiated tooth enamel}

$=\left\{v_{n}(H) \pm \frac{1}{2 h}\left[a+b\left(3 \cos ^{2} \gamma-1\right)\right]^{2}+\frac{9}{16} b^{2} \sin ^{2} 2 \gamma\right\}^{1 / 2}$

$\cos \gamma=\sin \theta \sin \theta_{n} \cos \varphi+\cos \theta \cos \theta_{n}$,

where $\gamma$ is the angle between magnetic field and $\mathbf{r}_{n}$ direction.

In our case $b<<v_{n}$, therefore, the expression (13) can be represented as a series expansion retaining only the first term:

$v_{0}^{ \pm}(H, \theta, \varphi)=\frac{g_{n} \beta_{n} H}{h} \pm$

$\frac{1}{2 h}\left\{a+b\left[3\left(\cos \left(\theta_{n}-\theta\right) \cos ^{2} \frac{\varphi}{2}+\cos \left(\theta_{n}+\theta\right) \sin ^{2} \frac{\varphi}{2}\right)^{2}-1\right]\right\}$

ENDOR signal defined by (11) is the spectrum averaged over the orientations of magnetic field at $\theta=$ const (see Fig. 1). If ENDOR frequencies are determined by (15) then the spectrum (11) consists of three doublets centered at $V_{n}$. Spectral positions of the doublets peaks are determined by:

$v_{1}^{ \pm}=v_{n} \pm \frac{1}{2 h}\left\{a+b\left[3 \cos ^{2}\left(\theta_{n}-\theta\right)-1\right]\right\}$,

$\gamma=\gamma_{\min }(\varphi=0)$

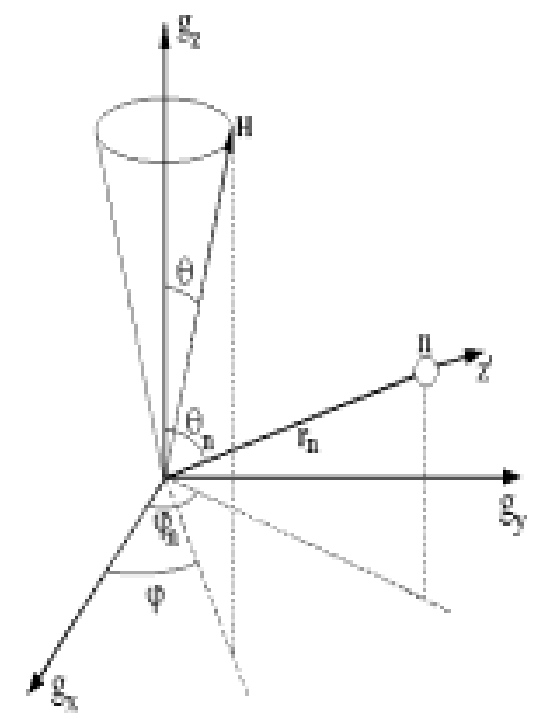

Fig. 1. Definition of the different polar angles describing the magnetic field vector and the direction of the interacting nucleus in the $g$-tensor axes frame. $z$ '-axial axis of the superhyperfine tensor.

$$
\begin{aligned}
& v_{2}^{ \pm}=v_{n} \pm \frac{1}{2 h}\left\{a+b\left[3 \cos ^{2}\left(\theta_{n}+\theta\right)-1\right]\right\} \\
& \gamma=\gamma_{\max }(\varphi=\pi)
\end{aligned}
$$

$v_{3}^{ \pm}=v_{n} \pm \frac{1}{2 h}(a-b), \gamma=\frac{\pi}{2}$

Note, that $v_{3}{ }^{ \pm}$is observed only for $\theta>\frac{\pi}{2}-\theta_{n}$. These expressions have been deduced from (11) assuming that the lineshapes are $\delta$-function-like for both EPR and ENDOR lines. Expressions (16)-(18) do not contain the relative intensities of ENDOR doublets and can be used to obtain superhyperfine parameters in the first-order approximation only. Their accurate values can be determined from computer fitting of the experimental spectrum and the one calculated on the base of (11) or (1). Such analysis has shown that the intensities of $v_{3}^{ \pm}$doublet exceed considerably the ones of other lines. Thus, $v_{3}{ }^{ \pm}$doublet determines ENDOR spectrum if the later takes place. This complicates the study of powder ENDOR because the $v_{3}^{ \pm}$doublet has no angular dependence.

It is convenient to introduce the value $\delta=v^{-}-v^{+}$ which does not depend on the shift of $v$ at $H$ scan and describes the orientation dependence of ENDOR spectrum.

\section{Experimental results}

\subsection{EPR}

EPR spectra of tooth enamel have been studied in detail, so we discuss only the data that will be necessary to understand ENDOR results.EPR spectrum observed by us in the unheated samples is determined mainly by the signal from $\mathrm{CO}_{2}^{-}$radicals. This signal has been well described by axial $g$-tensor with $g_{\perp}=2.0021, g_{\|}=1.9975$ and $g_{\|} \| c$ ( $c$ is the hexagonal axis of hydroxyapatite) that agrees with our earlier publication [10]. There was no reason to use a rhombic $\mathrm{g}$ tensor.

The sample annealing resulted in the decrease of the overall intensity of EPR spectrum. It can be explained by complete destruction of disordered $\mathrm{CO}_{2}^{-}$radicals [17] and particular disintegration of bulk $\mathrm{CO}_{2}^{-}$radicals. Besides, the relative intensities of weak signals changed slightly and the new spectrum components revealed. As to new signals, it should be noticed that EPR spectrum consists of seven components centered at $g=2.0003$ and separated by $2.18 \mathrm{mT}$. The central line of this signal is masked by more intensive signal from $\mathrm{CO}_{2}^{-}$radical. This spectrum has been assigned to $(\mathrm{CH} 3)-\mathrm{C}-\mathrm{R}$ radical activated in organic matter by annealing [11]. EPR spectrum 


\section{S. Ishchenko et al.: ENDOR study of irradiated tooth enamel}

at $g=2.006$ and $\Delta \mathrm{H}_{p p}=0.1 \mathrm{mT}$ was observed too. It has been attributed to $\mathrm{SO}_{2}^{-}$radical [26]. ENDOR signals caused by $\left(\mathrm{CH}_{3}\right)-\mathrm{C}-\mathrm{R}$ and $\mathrm{SO}_{2}^{-}$radicals were not observed. EPR spectra of irradiated tooth enamel before and after the annealing are shown in Fig. 2. The main EPR signal is seen to change slightly under the annealing.

\subsection{ENDOR}

ENDOR spectrum of unheated powder of tooth enamel has been recorded at $\mathrm{T}=77 \mathrm{~K}$ and $\mathrm{T}=4.2 \mathrm{~K}$. It consists of the singlet at Larmor frequency of ${ }^{31} \mathrm{P}$ nuclei and doublet at Larmor frequency of ${ }^{1} \mathrm{H}$ nuclei. The intensity of phosphorous line changes according to EPR lineshape while its linewidth does not vary at different magnetic fields within the experimental errors. Notice that field dependence of an ENDOR intensity has been called ENDOR-induced EPR (EI-EPR). The changing of ${ }^{1} \mathrm{H}$ doublet intensity distinguishes slightly from EPR lineshape on the lowfield wing of EPR line. Besides, ${ }^{1} \mathrm{H}$ doublet splitting increases considerably on the EPR line wings (Fig. 3). Similar behavior of the ${ }^{1} \mathrm{H}$ doublet field dependence has been also observed by Galtsev [19]. Reducing the measurement temperature from 77 to $4.2 \mathrm{~K}$ resulted in the increase of the overall intensity of ENDOR spectra only.

The doublet structure of the proton ENDOR signal vanished after annealing. However, a new structure was revealed for both ${ }^{1} \mathrm{H}$ and ${ }^{31} \mathrm{P}$ signals at liquid helium temperature and below. This structure was caused by interaction of $\mathrm{CO}_{2}^{-}$radical with nuclei of hydroxyapatite lattice. It is observed for all settings of magnetic field

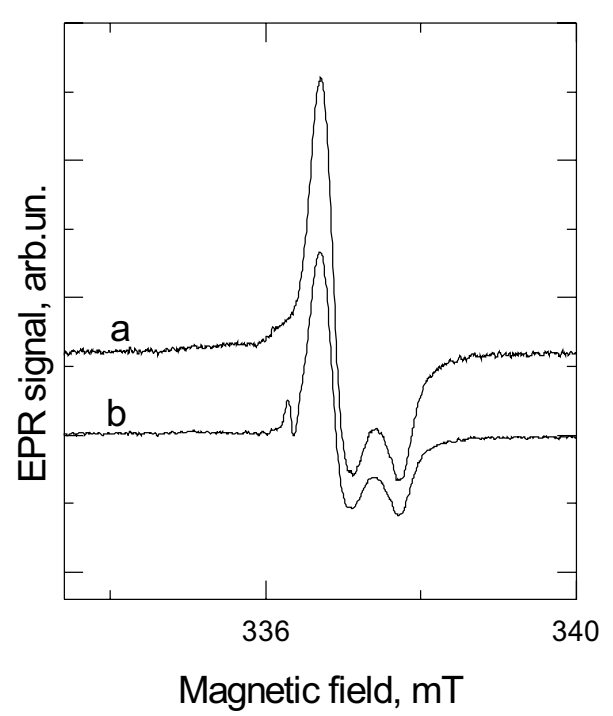

Fig. 2. EPR spectrum of irradiated tooth enamel powders near $g=2.0, \mathrm{~T}=300 \mathrm{~K}: \mathrm{a}$ - unheated sample, $\mathrm{b}-$ sample dried at $\mathrm{T}=250^{\circ} \mathrm{C}$ during 45 minutes.

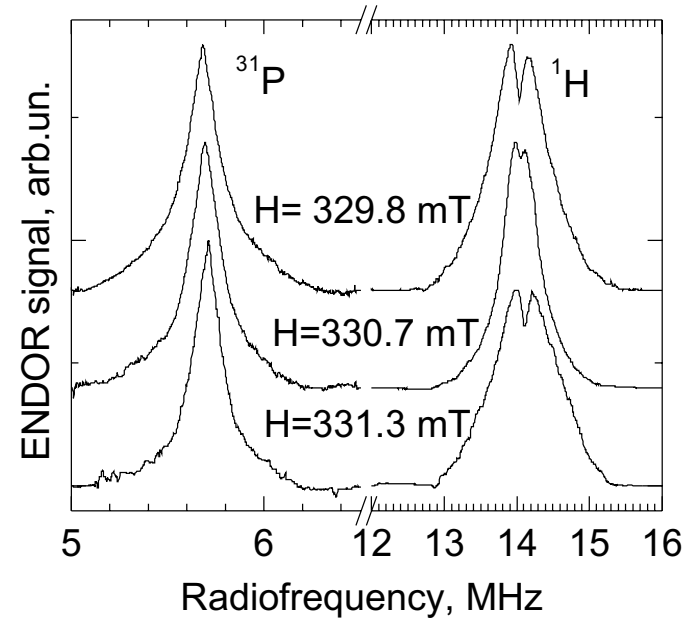

Fig. 3. ENDOR spectra of unheated sample of tooth enamel for different settings of magnetic field, $v_{m \omega}=9252 \mathrm{MHz}$.

within EPR spectrum and centered at Larmor frequencies of ${ }^{1} \mathrm{H}$ and ${ }^{31} \mathrm{P}$ nuclei.

The structure of ${ }^{31} \mathrm{P}$ ENDOR spectra consists of nine doublets (see Fig. 4). The $\delta$ values for all doublets are represented in Table I. The peaks of each ENDOR dou-

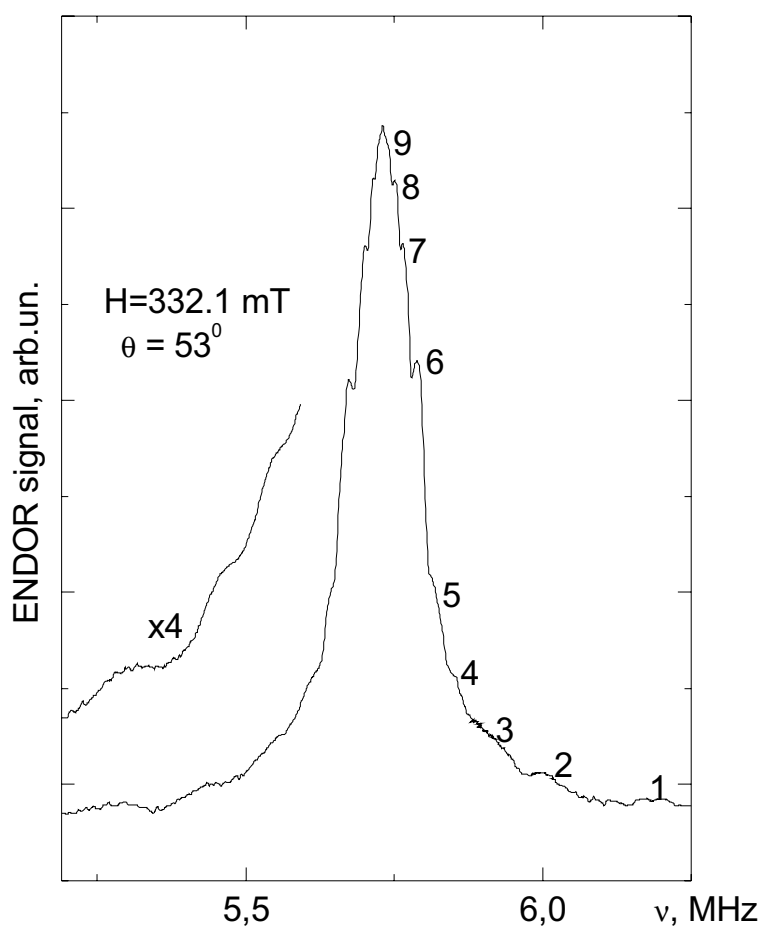

Fig. 4. ${ }^{31} \mathrm{P}$ ENDOR spectrum of annealed powder of tooth enamel. ENDOR doublets caused by nuclei of different shells are labeled by $1-9$. 


\section{S. Ishchenko et al.: ENDOR study of irradiated tooth enamel}

Table $\mathrm{I}^{31} \mathrm{P}$ superhyperfine coupling constants of the $\mathrm{CO}_{2}^{-}$radical in tooth enamel.

\begin{tabular}{|c|c|c|c|c|c|c|c|c|c|}
\hline Doublet & 1 & 2 & 3 & 4 & 5 & 6 & 7 & 8 & 9 \\
\hline$\delta, \mathrm{kHz}$ & $\begin{array}{l}920- \\
860\end{array}$ & 530 & $380-320$ & 220 & 170 & $130-110$ & $60-40$ & $40-30$ & 11 \\
\hline$a / \mathrm{h}, \mathrm{kHz}$ & $\begin{array}{l}-310 \\
\pm \\
\pm\end{array}$ & $\begin{array}{l}-165 a \\
+20\end{array}$ & $-90_{ \pm} 20$ & $\begin{array}{l}-49 a \\
+15\end{array}$ & $\begin{array}{l}-33 a \\
+10\end{array}$ & $-20_{ \pm} 10$ & $0_{ \pm} 10$ & 0 & \\
\hline$b / \mathrm{h}, \mathrm{kHz}$ & $\begin{array}{l}630 \\
\pm 20\end{array}$ & $385 \pm 20$ & $260 \pm 20$ & $183 \pm 15$ & ${ }^{\frac{T}{14}} \pm 10$ & $100 \pm 10$ & $60 \pm 10$ & $56-38^{b}$ & \\
\hline$\theta_{\mathrm{n}}, \mathrm{deg}$ & $28_{ \pm} 4$ & $90_{ \pm} 4$ & $40_{ \pm} 4$ & $90_{ \pm} 4$ & $90 \pm 4$ & $60 \pm 4$ & $65 \pm 4$ & & \\
\hline $\mathbf{r}_{n}, \mathrm{~nm}$ & $\begin{array}{l}0 . \overline{37} \\
\pm 0.01\end{array}$ & $\begin{array}{l}0 . \overline{44} \\
\pm 0.03\end{array}$ & $\begin{array}{l}0 . \overline{50} \\
\pm^{0.01}\end{array}$ & $\begin{array}{l}0 . \overline{56} \\
\pm 0.03\end{array}$ & $\begin{array}{l}0.6 \overline{0} \\
\pm^{0.03}\end{array}$ & $\begin{array}{l}0.6 \overline{8} \\
\pm 0.01\end{array}$ & $\begin{array}{l}0.8 \overline{1} \\
\pm 0.01\end{array}$ & & \\
\hline$\theta_{n}^{l}, \operatorname{deg}$ & 33 & 90 & $\overline{4} 7$ & 90 & 90 & 59 & 65 & & \\
\hline $\mathbf{r}_{n}^{l}, \mathrm{~nm}$ & 0.41 & 0.47 & 0.50 & 0.55 & 0.63 & 0.68 & 0.80 & $\begin{array}{l}0.83- \\
0.94\end{array}$ & 0.97 \\
\hline shell & I & II & III & IV & $\mathrm{V}$ & VI & VIII & IX-XIV & $\mathrm{XV}$, \\
\hline
\end{tabular}

$a$ - values calculated with using the expression (19)

$b$ - values calculated with using the expression (20) and $\mathrm{r}_{n}^{l}$

blet can be formed by nuclei of one shell as well as by the contributions of nuclei involved into several shells. The structure of ${ }^{31} \mathrm{P}$ ENDOR spectra can be arbitrarily subdivided on a three groups of lines. The first group contains the doublets $1,3,6,7$ which depends on setting of the magnetic field. The second group involves doublets $2,4,5$. Their spectral positions do not shift when a setting of the magnetic field is changed. Finally, the lines being near Larmor frequency are attributed to the third group. These are 8 and 9 doublets; their lineshapes and intensities are modified at different settings of the magnetic field. But their spectral shifts are small and can not be determined accurately.

The structure of ${ }^{1} \mathrm{H}$ ENDOR spectra is less distinctive than the one ${ }^{31} \mathrm{P}$ although seven doublets can be exuded. The typical ${ }^{1} \mathrm{H}$ ENDOR spectrum is represented in Fig. 5. Its lineshape is modified while the setting of the magnetic field is changed, however, the shifts of the peaks can not be determined accurately. The values of $\delta$ are represented in Table II. Note that intensive structureless signal centered at $v_{\mathrm{n}}$ revealed in both ${ }^{1} \mathrm{H}$ and ${ }^{31} \mathrm{P}$ ENDOR spectra too. It can be attributed to centers located in sites with high concentration of defects. The contribution from distant nuclei is also possible.

\section{Analysis of the spectra and discussion}

The admixed hydrogen atoms other than ones involved into hydroxyapatite lattice are present in unheated tooth enamel. They occur in crystallites in the forms of $\mathrm{H}_{2} \mathrm{O}$ molecules, admixed $\mathrm{OH}$ groups and $\mathrm{H}$-centers of different types. These atoms form the large amounts of hy- droxyapatite defects that lead to disappearance of superhyperfine structure in ENDOR spectra. Besides, the hydrogen arranged randomly appears to be responsible for

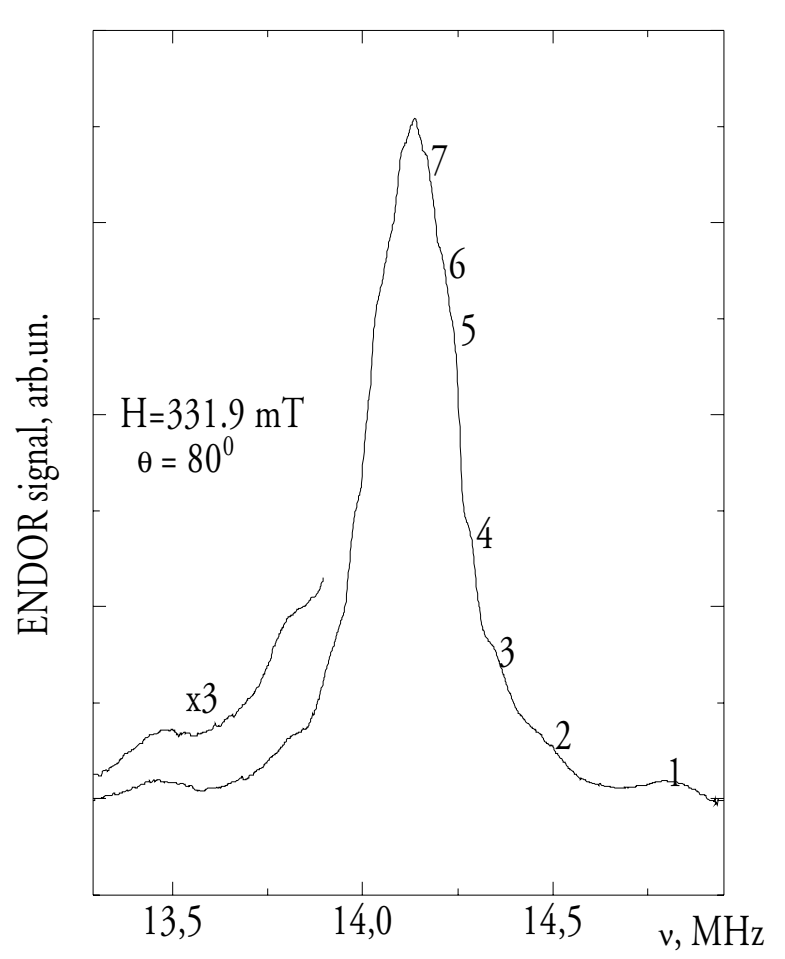

Fig. 5. ${ }^{1} \mathrm{H}$ ENDOR spectrum of annealed powder of tooth enamel. ENDOR doublets caused by nuclei of different shells are numbered by $1-7$. 


\section{S. Ishchenko et al.: ENDOR study of irradiated tooth enamel}

the doublet structure of proton ENDOR signal. The similar spectrum lineshape has been described in the work [24] devoted to matrix ENDOR. The peaks positions of such spectrum are determined by competition between signal increase from distant nuclei due to the growth of their amounts and signal decrease due to the diminution of the transition probabilities and the relaxation mechanisms.

The change of the doublet splitting of the proton ENDOR (Fig. 3) and deviation of EI-EPR lineshape from EPR one can be explained by the presence in the ENDOR signal of two components corresponding to two paramagnetic centers. The first component is a narrow intensive doublet with the splitting $\Delta_{1}=100-140 \mathrm{kHz}$. It dominates in the central part of the EPR spectrum. The value of $\Delta_{1}$ is changed within the mentioned region for different samples but it is constant for a certain sample between $g=1.9975$ and $g=2.0021$. Thus, the narrow component can be attributed to the main EPR signal caused by the bulk $\mathrm{CO}_{2}^{-}$radical. The second component is the wider doublet with splitting $\Delta_{2}=260 \mathrm{kHz}$. It dominates on the wings of the EPR spectrum. The nature of a center that causes this signal is not clear. The abovementioned disordered $\mathrm{CO}_{2}^{-}$radical is probably responsible for it. The relaxation characteristics of the disordered radicals appear to be more favourable for ENDOR observations. This increases the contribution of these centers in EI-EPR signal and results in different lineshapes of EPR and EI-EPR spectra. Such relaxation amplification of ENDOR signal is probably absent in phosphorous ENDOR. ENDOR spectra of the annealed tooth enamel samples have been analyzed using the theory of powder ENDOR. The $a, b$ and $\theta_{n}$ parameters have been estimated using (16) - (18) and the assumption that $\theta=\left[\left(g^{2}-g_{\perp}^{2}\right) /\left(g_{\|}^{2}-g_{\perp}^{2}\right)\right]^{1 / 2} \quad$ where $g=h v_{m \omega} / \beta H$ at $H=H_{0}$. Accurate values of parameters have been determined by computer fitting of the spectra calculated according to (11) to experimental ones. In these simulations the gaussian with $\Delta H=0.25 \mathrm{mT}$ and lorentzian with $\Delta v=10-30 \mathrm{kHz}$ for different shells have been used as EPR and ENDOR lineshapes, respectively.

Note that parameters variation did not resulted in the accurate agreement between calculated and experimental dependencies. Experimental curves were more smooth than the calculated ones. The rhombic g-tensor with $\mathrm{g}_{x}=2.0030, \mathrm{~g}_{y}=2.0015, \mathrm{~g}_{z}=1.9970$ represented in [16] and the expression (1) have been used to simulate the ENDOR spectra, too, but improvement has not been obtained. Thus, the mentioned discrepancy appears to be due to the neglection of relaxation orientation dependencies in (1) and (11).

The analysis of the expressions (16)-(18) and (11) has shown that angular variation of the second group (doublets 2, 4, 5) of phosphorous ENDOR is characteristic for nuclei with $\theta_{n}=90^{\circ}$. Powder ENDOR spectra of such nuclei consist of dominating doublets (18) which have no orientation dependencies. In this case the value $|a-b|$ can be determined at once from the experiment. To estimate the constants $a$ and $b$ separately the following approximation can be made:

$a=a_{0} e^{-k r}$,

where $a_{0}$ and $\mathrm{k}$ are the parameters which can be determined using the expression (19) for nuclei of first group (doublets 1, 3, 6, 7).

The anisotropic superhyperfine interaction has been considered as pure dipole-dipole interaction. Then constant $b$ can be written as:

$b=\frac{g \beta g_{n} \beta_{n}}{r^{3}}$,

where $r \equiv \mathbf{r}_{n}$ corresponds to the distance between $\mathrm{CO}_{2}$ radical site and nucleus $n$.

The values $\theta_{n}$ and $\mathbf{r}_{n}$ obtained from ENDOR spectra for different nuclei allow to determine the location of $\mathrm{CO}_{2}^{-}$ radical in crystal lattice. The values $\theta_{n}^{l}$ and $\mathbf{r}_{n}$ for different possible sites of $\mathrm{CO}_{2}^{-}$in hydroxyapatite lattice have been calculated using the model of its structure (Ref. 28) and compared with $\theta_{n}$ and $\mathbf{r}_{n}$. It has been found that only $\mathrm{CO}_{2}^{-}$in $\mathrm{B}$ site (phosphorous substitution) at $g_{\|} \| c$ agrees well with the experimental data. Some discrepancy between experimental and lattice data for phosphorous nuclei of shell I (see Table I) can be explained by their shift towards the defect. Note that the ENDOR lines due to ${ }^{31} \mathrm{P}$ nuclei of VII shell were not observed. In accordance with the computer simulation these lines have intense angular dependencies and they are broadened strongly. Besides, they are likely superimposed with lines of shell V and VI.

A constant $a$ can be considered to be equal to zero for phosphorous of VIII shell and more distant nuclei. Then the frequency region for lines caused by these nuclei can be estimated by means of (15) and (20) using lattice data for $\theta_{n}^{l}$ and $\mathbf{r}_{n}^{l}$. Such calculations permit to explain the peaks 8,9 in phosphorous ENDOR (see Table I).

The values $\theta_{n}^{l}$ and $\mathbf{r}_{n}^{l}$ have been also used to simulate proton ENDOR spectra. Constants $b$ have been calculated from $\mathbf{r}_{n}^{l}$. Constants $a$ have been determined by computer fitting of calculated ENDOR spectra to experimental ones. The obtained values of $a$ have not been contradicted with the model of center when $\mathrm{OH}$ vacancy is situated in the nearest surrounding of $\mathrm{CO}_{2}^{-}$ radical. This finding agrees with the well-known model [29] of carbon incorporation in hydroxyapatite structure:

$\mathrm{Ca}^{2+}+\mathrm{PO}_{4}^{3-}+\mathrm{OH}^{-} \leftrightarrow \mathrm{V}_{\mathrm{Ca}}+\mathrm{CO}_{3}^{3-}+\mathrm{V}_{\mathrm{OH}}$,

where $\mathrm{CO}_{3}{ }^{3-}$ molecular ion is a precursor of $\mathrm{CO}_{2}^{-}$radical.

The $\mathrm{CO}_{2}^{-}$site and surrounding apatite structure are represented in Fig. 6-8. The neighbour nuclei ${ }^{31} \mathrm{P}$ and ${ }^{1} \mathrm{H}$ are numbered in accordance with Tables I, II. 


\section{S. Ishchenko et al.: ENDOR study of irradiated tooth enamel}

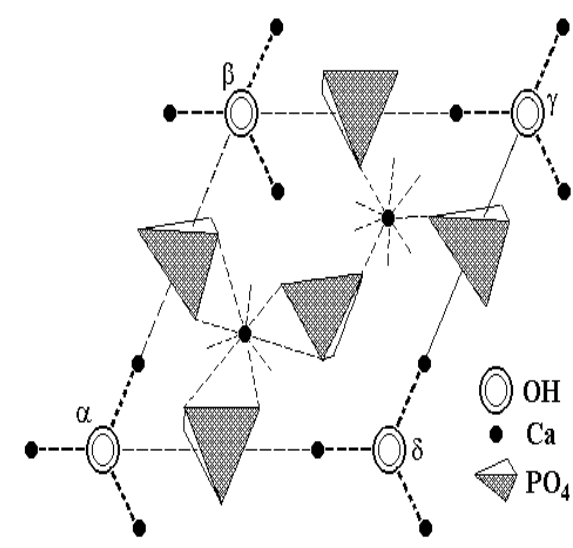

Fig. 6. Fragment of hydroxyapatite structure. $\alpha, \beta, \gamma, \delta$ are the hexagonal axes.

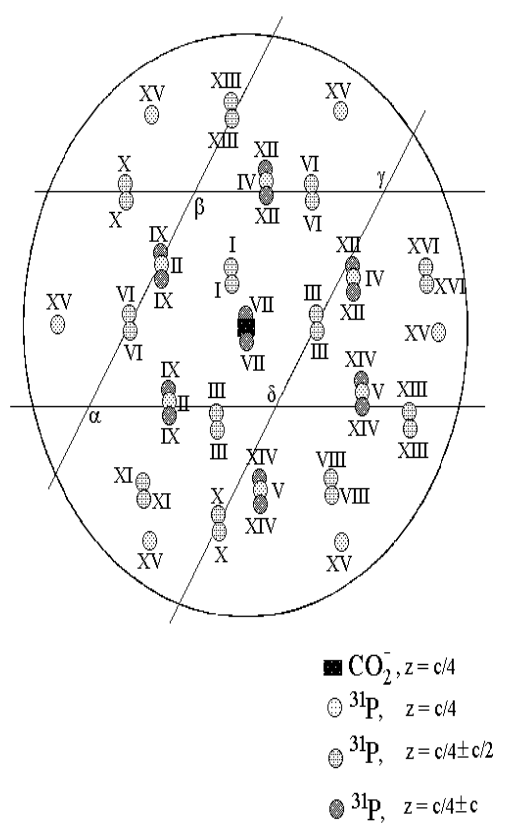

Fig. 8. The locations of phosphorous nuclei of different shells relative to $\mathrm{CO}_{2}^{-}$radical.

\section{Conclusion}

The signal caused by $\mathrm{CO}_{2}{ }^{-}$dominates in EPR spectrum of irradiated tooth enamel. The contributions of other centers have been revealed too, but their intensities are smaller. Two types of $\mathrm{CO}_{2}^{-}$radicals are observed in unheated samples. One of them is the bulk center. It causes the angular variations of EPR spectrum in a tooth plate. The centers of the second type are disordered and cause EPR spectrum of powder line shape. They appear to be located on the surface of crystallites.

The singlet at Larmor frequency of ${ }^{31} \mathrm{P}$ nuclei and doublet at Larmor frequency of ${ }^{1} \mathrm{H}$ nuclei are observed in ENDOR spectrum of unheated tooth enamel. The
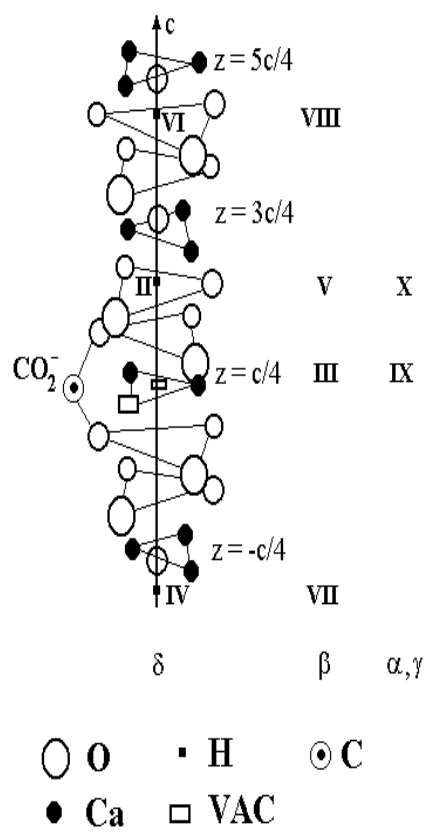

Fig. 7. Hydroxyapatite structure close to the hexagonal c-axis and proposed site for $\mathrm{CO}_{2}^{-}$radical. The numbers $\mathrm{I}-\mathrm{X}$ are shown the location of protons of different shells.

value of doublet splitting depends on the settings of magnetic field and is equal to $100-140 \mathrm{kHz}$ and $260 \mathrm{kHz}$ in the central part and wings of EPR line, respectively. The doublet structure of proton ENDOR can be explained by the theory of matrix ENDOR assuming the presence of a large amount of admixed hydrogen atoms. The modification of proton ENDOR line at different settings of magnetic field appears to be connected with the existence of two types of $\mathrm{CO}_{2}^{-}$radicals. These radicals cause the proton ENDOR signal with various splittings, but their contributions are different in the central part and on the wings of EPR line.

The annealing at $250{ }^{\circ} \mathrm{C}$ results in the decrease of the overall intensity of EPR spectrum due to complete destruction of the disordered $\mathrm{CO}_{2}^{-}$radicals and particular disintegration of the bulk $\mathrm{CO}_{2}{ }^{-}$radicals. Small variation of EPR lineshape due to the redistribution of the intensities of weak signals and appearance of the new signal from centers activated by annealing is also observed. The structure of phosphorous and proton ENDOR centered at Larmor frequencies of ${ }^{1} \mathrm{H}$ and ${ }^{31} \mathrm{P}$ nuclei reveals after annealing. Its analysis allows to determine the constants of superhyperfine interaction of $\mathrm{CO}_{2}^{-}$radical with neighbour nuclei and to conclude that $\mathrm{CO}_{2}^{-}$ion substitutes phosphorous in hydroxyapatite lattice of tooth enamel (B site). This substitution is found to be accompanied by the creation of $\mathrm{OH}$ vacancy in the nearest surroundings and shift of phosphorous nuclei of shell I towards the 
S. Ishchenko et al.: ENDOR study of irradiated tooth enamel

Table II. ${ }^{1} \mathrm{H}$ superhyperfine coupling constants of the $\mathrm{CO}_{2}^{-}$radical in tooth enamel.

\begin{tabular}{|c|c|c|c|c|c|c|c|}
\hline Doublet & 1 & 2 & 3 & 4 & 5 & 6 & 7 \\
\hline $\begin{array}{l}\delta, \mathrm{kHz} \\
\theta_{n}^{l}, \mathrm{deg} .\end{array}$ & $\begin{array}{c}1350 \\
60\end{array}$ & 650 & $\begin{array}{c}370 \\
78,37\end{array}$ & $\begin{array}{c}280 \\
70,33\end{array}$ & $\begin{array}{c}185-175 \\
51\end{array}$ & $\begin{array}{c}140 \\
46,81,75\end{array}$ & 62 \\
\hline $\mathbf{r}_{n}^{l}, \mathrm{~nm}$ & 0.42 & & 0.60 & $0.62,0.67$ & 0.75 & $0.81,0.81,0.83$ & $0.9, \ldots$ \\
\hline$b / h \sim 1 / r_{n}^{3}, \mathrm{kHz}$ & 1070 & & 367 & 332 & 188 & $155,149,138$ & \\
\hline$a / \mathrm{h}, \mathrm{kHz}$ & $\begin{array}{l}-280 \\
\pm 30\end{array}$ & & $\begin{array}{l}-20 \\
\pm 20\end{array}$ & $\begin{array}{c}0 \\
\pm 20\end{array}$ & $\begin{array}{c}0 \\
\pm 20\end{array}$ & $\begin{array}{c}0 \\
\pm\end{array}$ & 0 \\
\hline shell & II & (III-IV) & III-V(VI) & $\begin{array}{c}\mathrm{V}, \mathrm{VI},(\mathrm{VIII}- \\
\mathrm{IX})\end{array}$ & VII & VIIİ,IX,X & $\mathrm{XI}, \ldots$ \\
\hline
\end{tabular}

defects by $d r=0.04 \mathrm{~nm}$. The experimental angular dependencies of ENDOR lines are more smooth than the calculated ones. This discrepancy appears to be due to the neglection of an orientational dependence of relaxation times in the theory. All EPR and ENDOR spectra have been described by axial $g$-tensor with $g_{\|}=$ $=1.9975$ and $g_{\perp}=2.0021$ at $g_{\|} \| c$.

\section{Acknowledgments}

The authors wish to thanks Prof. A. B. Roitsin for consultations on the theory of powder ENDOR and Dr. S. V. Virko for assistance in computer calculations.

\section{References}

1. M. A.Scott, H. G.Hallacher, T. P. J. Han, B. Henderson, Radiat.Eff.Defect S., 136, p.957 (1995).

2. E. Borovsky, Terapevticheskaya Stomatologiya, Meditsina, Moscow, 1988 (in Russian).

3. J. Tatsumi-Miyajima, Nucl.Instr.\&Meth., A257, p.417 (1987).

4. M. Ikeya, Appl.Radiat.Isot., 44, p.1(1993).

5. A. B. Brik, S. S. Ishchenko, L. G. Rosenfeld, D. I. Zabolotnii, I. S. Zaritskaya, Med.Radiology, p.25(1993).

6. R. Grun, Radiat.Isot., 40, p.1045 (1989).

7. B. A. Blackwell, H. P. Schwarcz, Appl.Radiat.Isot., 44,p. 243 (1993).

8. K. Sato, Calcif.Tissue Int., 29, p.95 (1979).
9. Y. Doi, T. Aoba, M. Okazaki, J. Takahashi, Y. Moriwaki, Calcif. Tissue Int., 28, p.107 (1979).

10. S. S. Ishchenko, S. M. Okulov, I. P. Vorona, A. B. Roitsin, A. A. Klimov, Sov. Phys. Solid State, 34, p.1522 (1992).

11. G. Baquet, V. Q. Truong, M. Vignoles, J. C. Trombe, G. Bonel, Calcif. Tissue Int., 33, p.105 (1981).

12. F. J. Callens, R. M. H. Verbeeck, P. F. A. Matthys, L. C. Martens, E. R. Boesman, Calcif. Tissue Int., 41, p.124 (1987).

13. F. J. Callens, Nucleonika, 42, p.565 (1997).

14. P. Moens, F. Callens, S.V an Doorslaer, P. Matthys, Phys. Rev., B53, p.5190 (1996).

15. T. Shimano, M. Iwasaki, C. Miyazawa, T. Miki, A. Kai, M. Ikeya, Appl.Radiat.Isot., 40, p.1035 (1989).

16. P. D. Moens, F. J. Callens, P. F. Matthys, J. Chem. Soc. Faraday Trans., 90, p.2653 (1994).

17. F. Callens, P. Moens, P. Verbeeck, Calcif.Tissue Int., 56, p.543 (1995).

18. H. van Willigan, A. H. Roufosse, M. J. Glimcher, Calcif.Tissue Int., 31, p.70 (1980).

19. V. E. Galtsev, Appl. Radiat. Isot., 47, p.1365 (1996).

20. G. Rist, J. Hyde, J. Chem.Phys., 52, p.4633 (1970).

21. G. C. Hurst, T. A. Henderson, R. W. Kreilick, J.Am.Chem.Soc., 107, p.7294 (1985).

22. P. A. Narayana, M. K. Bowman, D. Becker, L. Kevan, J.Chem.Phys., 67, p.1990 (1977).

23. B. M. Hoffman, R. A. Venters, J. Martinsen, J.Magn.Res., 62, p.537 (1985).

24. L. Kevan, S. Schlick, K. Torlyama, M. Iwasaki, J.Phys.Chem, 84, p.1950 (1980).

25. V. G. Grachev, M. F. Deigen,Usp. fiz. nauk, 125, p.631 (1978).

26. D. U. Schramm, A. M. Rossi, Appl.Radiat.Isot, 47, p.1443 (1996).

27. J. R. Niklas, J.-M. Spaeth, Phys.Stat.Sol.(b), 101, p.221 (1980).

28. M. I. Kay, R. A. Young, A. S. Posner, Nature, 204, p.1050 (1964).

29. J. C. Larbarthe, M. Therasse, G. Bonel, G. Montel, hebd. Seanc.Acad.Sci.Paris, C, 276, p.1175 (1973). 